\title{
Efficient Synthesis of Azide-bearing Cofactor
}

Mimics

Lindsay R. Comstock and Scott R. Rajski*

University of Wisconsin-Madison

School of Pharmacy

777 Highland Ave.

Madison, WI 53705

Email: srrajski@pharmacy.wisc.edu

\section{Table of Contents}

General Experimental Methods $\quad$ S2

${ }^{1} \mathrm{H}$ NMR Spectrum of $\mathbf{1 0} \quad$ S3

${ }^{13} \mathrm{C}$ NMR Spectrum of $\mathbf{1 0} \quad$ S4

${ }^{1} \mathrm{H}$ NMR Spectrum of $\mathbf{1 1} \quad$ S5

${ }^{13} \mathrm{C}$ NMR Spectrum of $\mathbf{1 1} \quad$ S6

$\begin{array}{ll}{ }^{1} \mathrm{H} \text { NMR Spectrum of } \mathbf{1 2} & \text { S7 }\end{array}$

${ }^{13}$ C NMR Spectrum of $12 \quad$ S8

${ }^{1} \mathrm{H}$ NMR Spectrum of $\mathbf{1 3} \quad$ S9

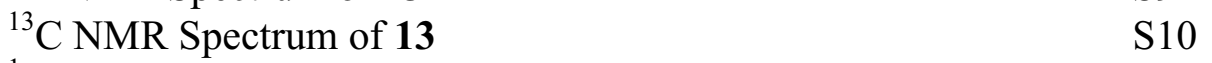

${ }^{1} \mathrm{H}$ NMR Spectrum of $\mathbf{1 5} \quad \mathrm{S} 11$

${ }^{13}$ C NMR Spectrum of $\mathbf{1 5} \quad \mathrm{S} 12$

${ }^{1} \mathrm{H}$ NMR Spectrum of $\mathbf{1 6} \quad \mathrm{S} 13$

${ }^{13} \mathrm{C}$ NMR Spectrum of $\mathbf{1 6} \quad \mathrm{S} 14$

${ }^{1}$ H NMR Spectrum of $6 \quad \mathrm{~S} 15$

${ }^{13} \mathrm{C}$ NMR Spectrum of $6 \quad \mathrm{~S} 16$ 
General Experimental Methods. All reactions were carried out under an inert atmosphere of argon unless indicated otherwise. All reagents were obtained from commercial suppliers and used as received unless otherwise noted. The silica gel used in column flash chromatography was Merck no. 9385, 60 A, 230-400 mesh. Analytical TLC was conducted on EM Science silica gel plates with detection by ninhydrin and/or UV light. Tetrahydrofuran (THF) was distilled over Na and benzophenone; Toluene was distilled over $\mathrm{Na}$. 

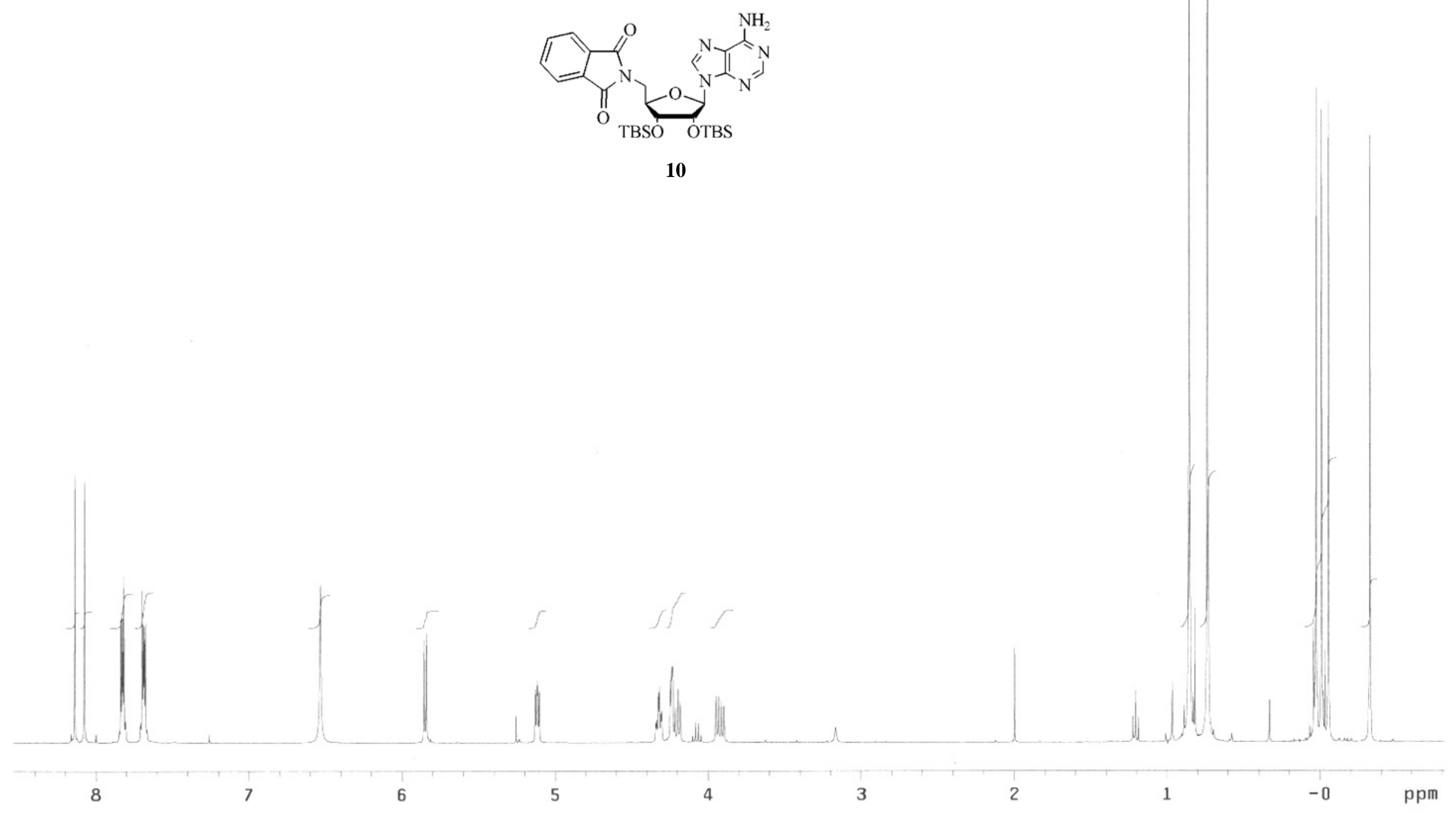


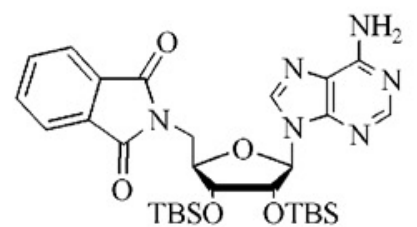

10

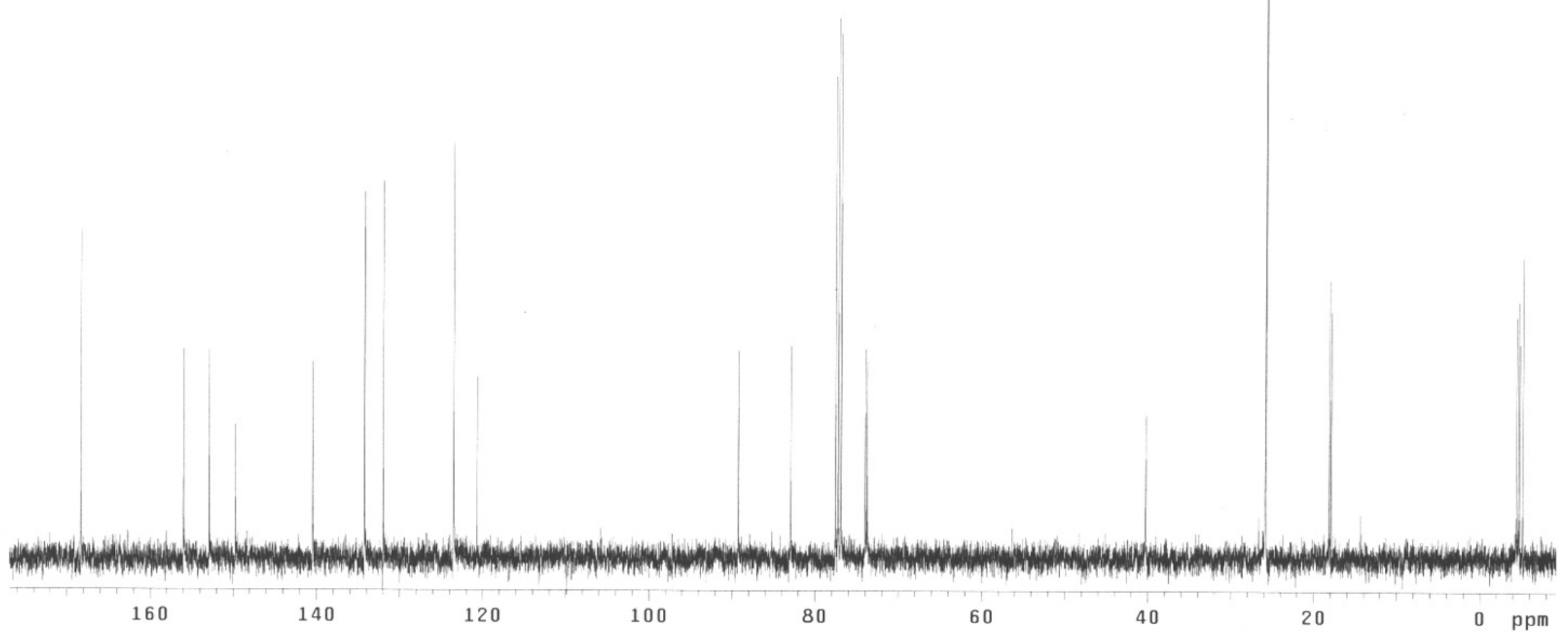



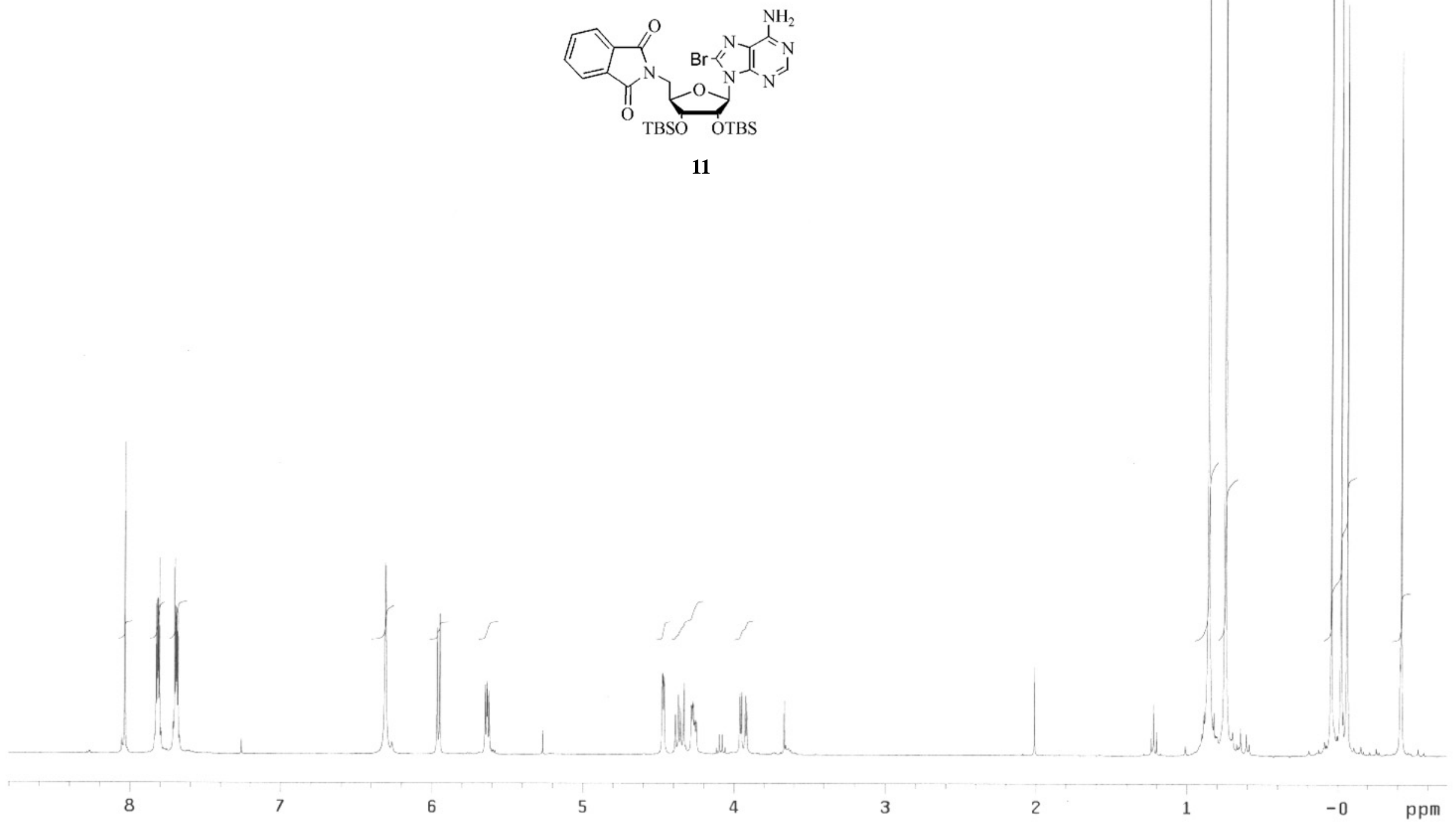


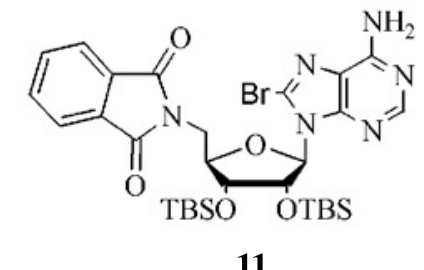

11

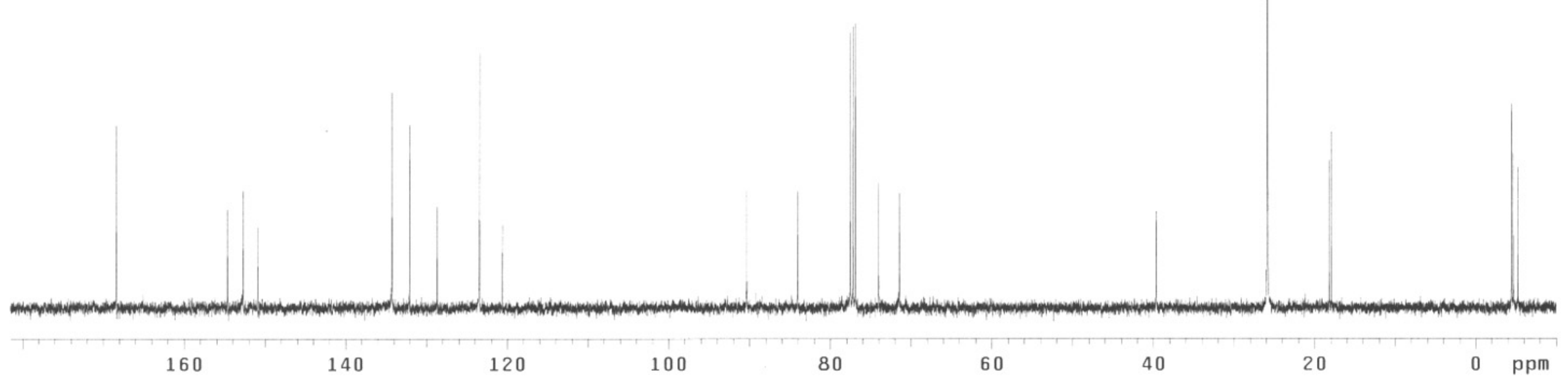



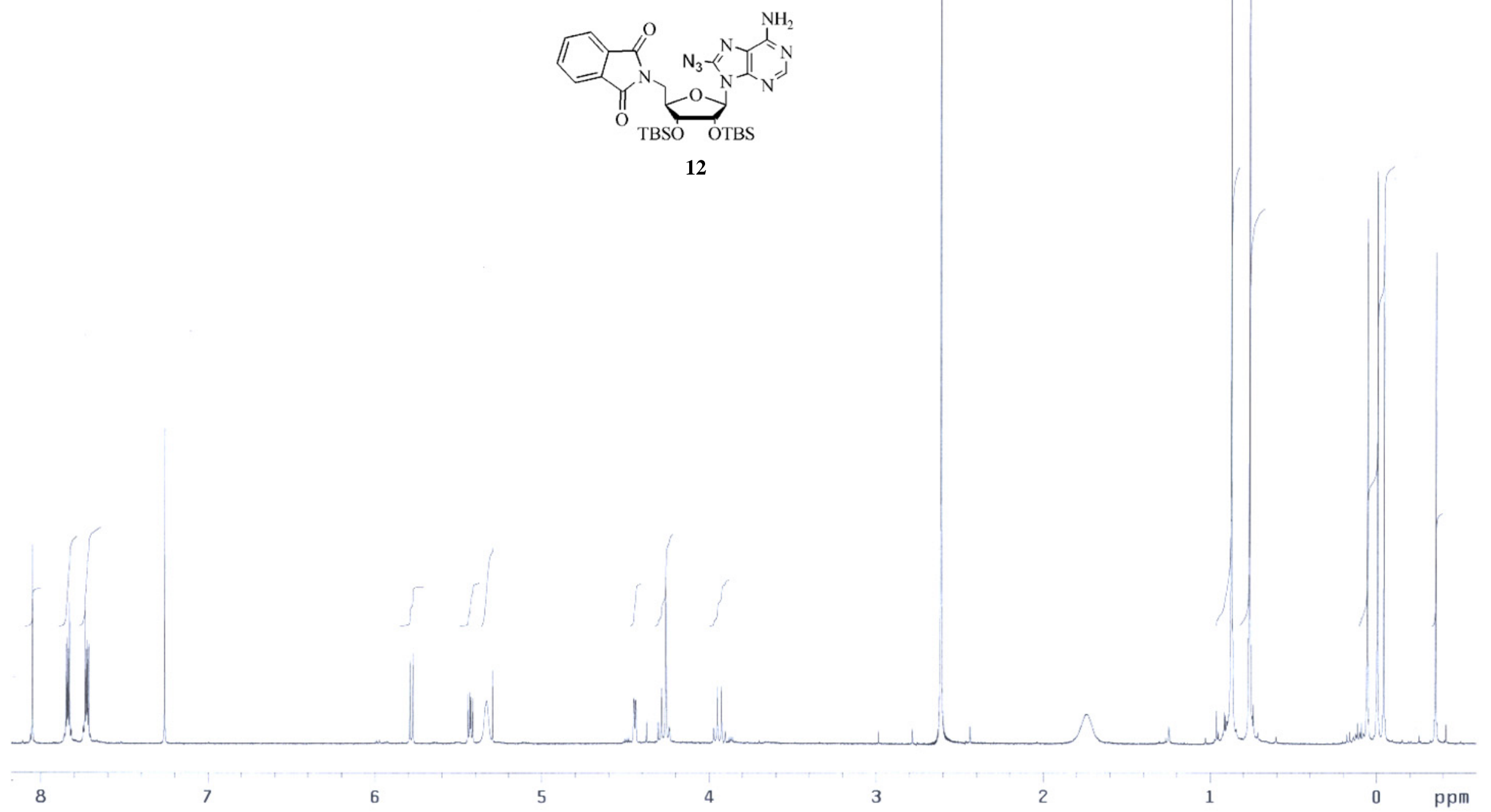


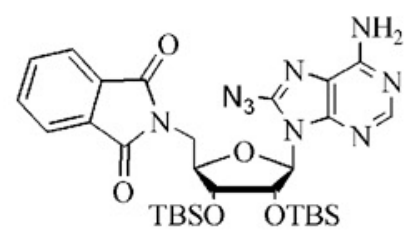

12

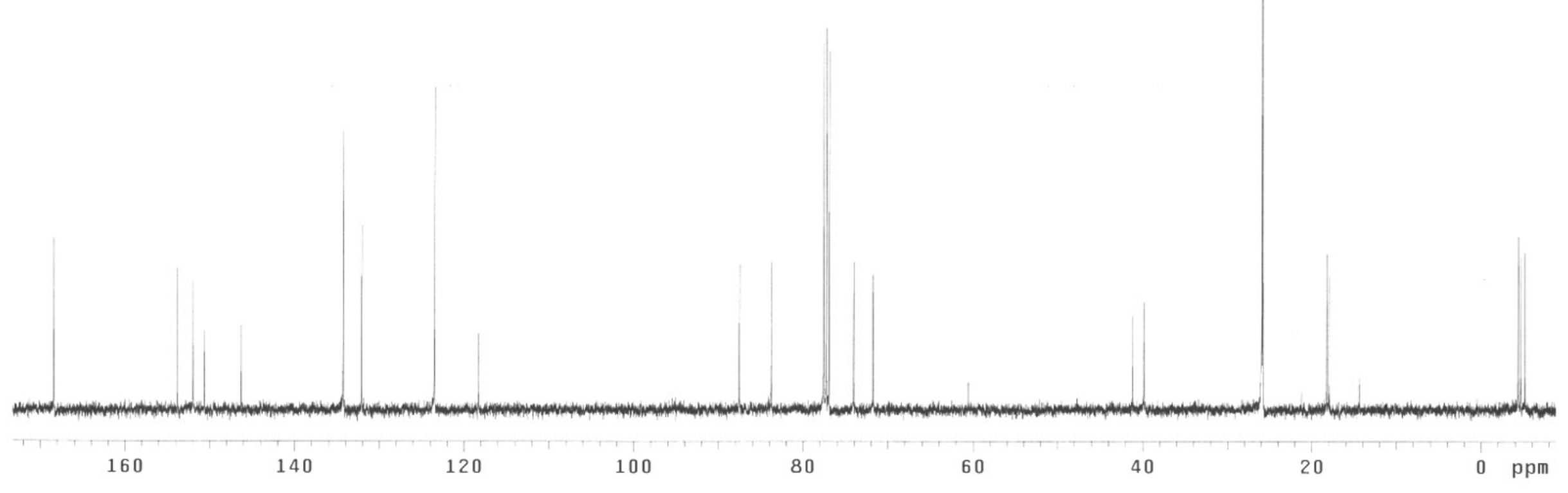




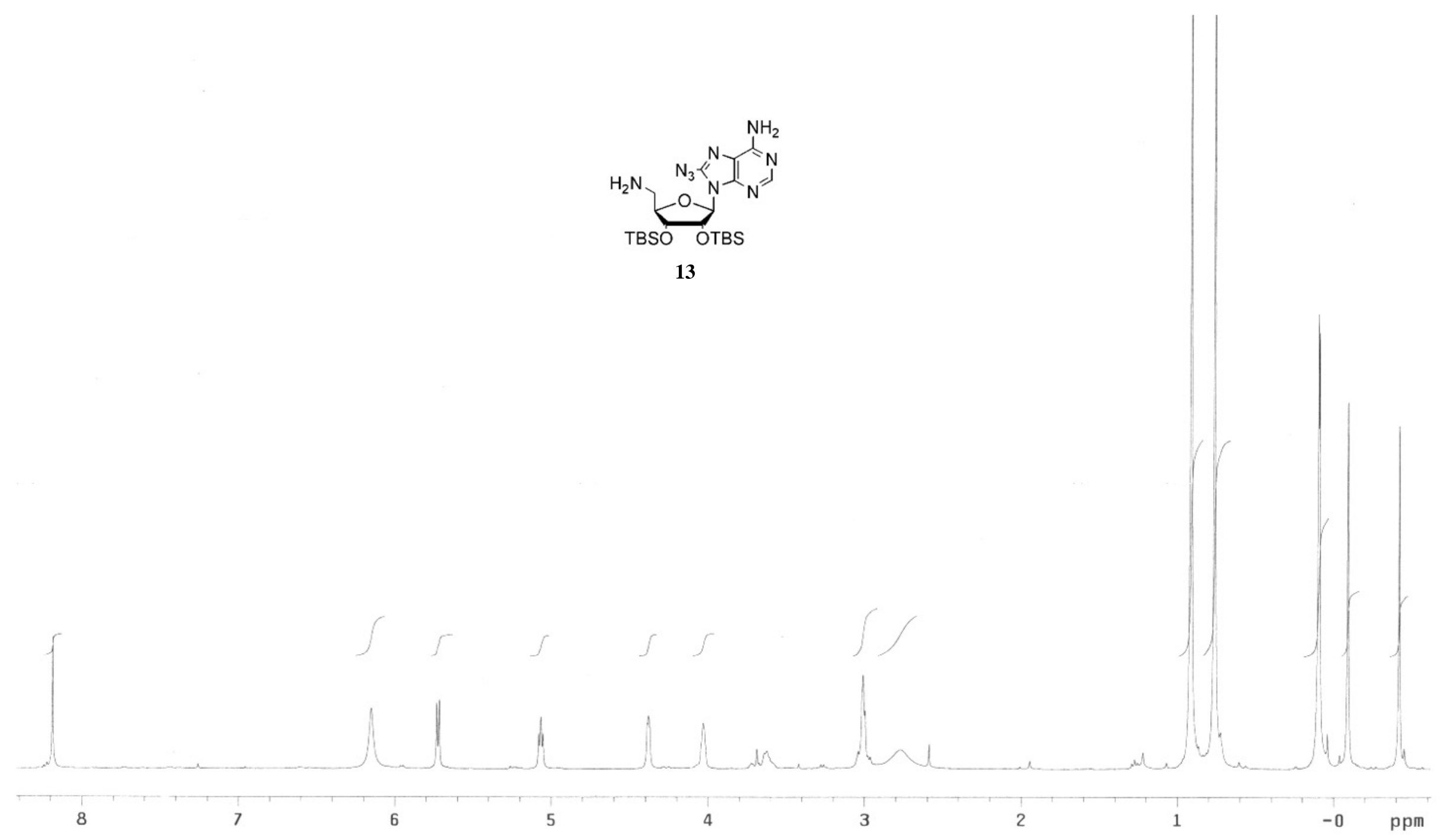




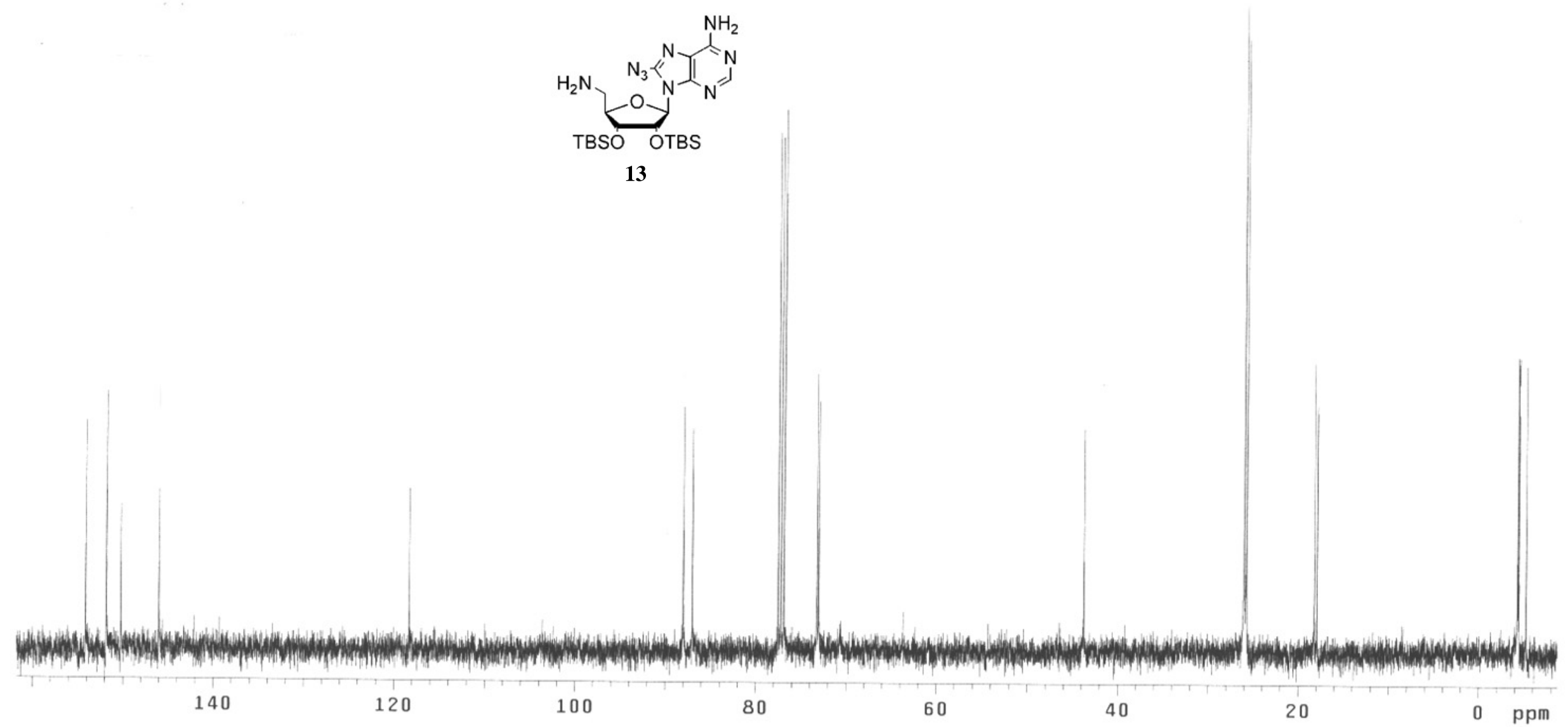




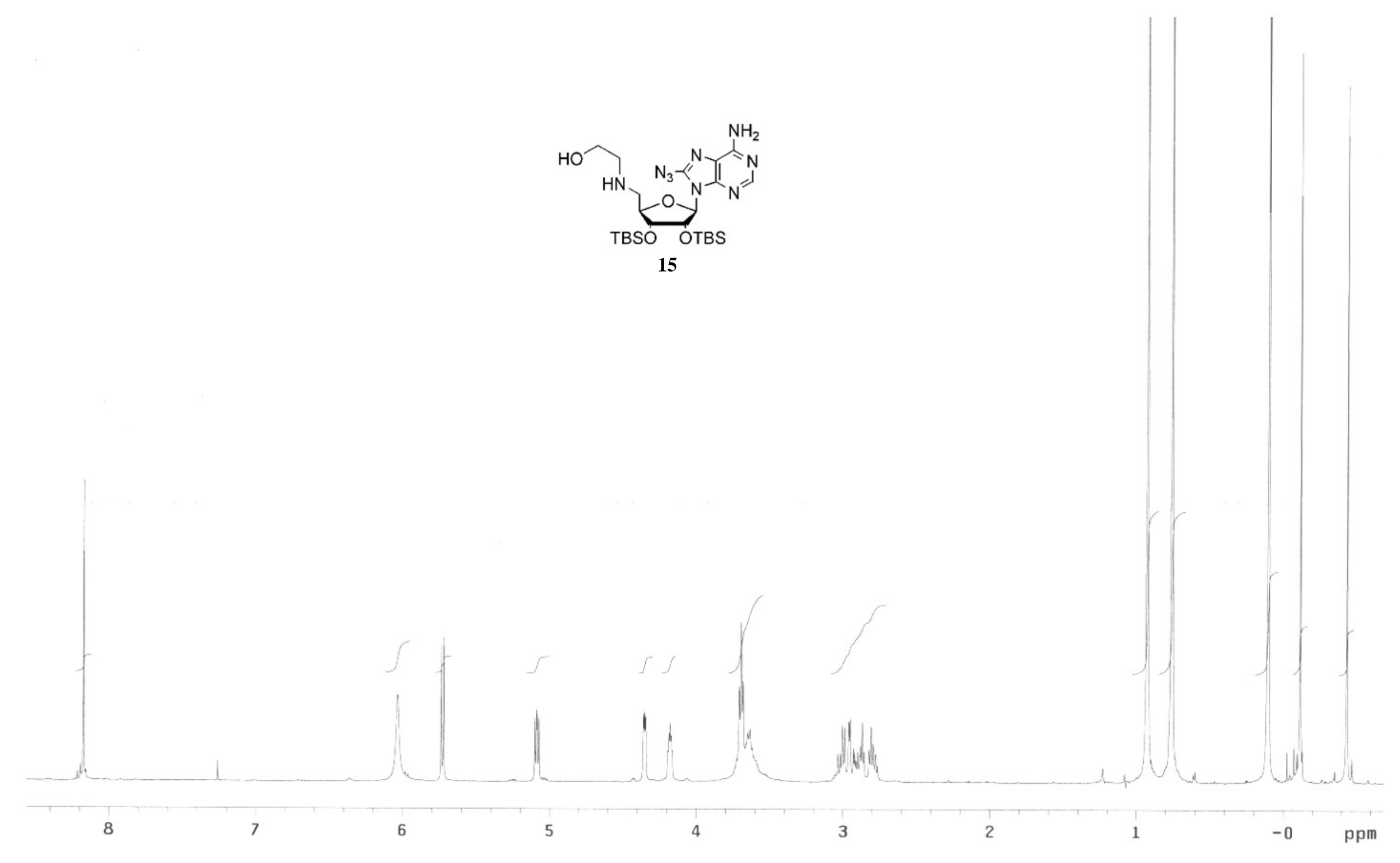




$$
\text { TBSO OTBS }
$$

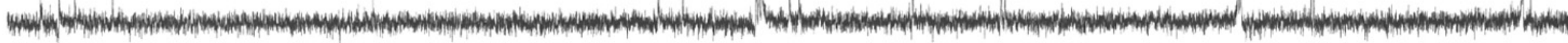




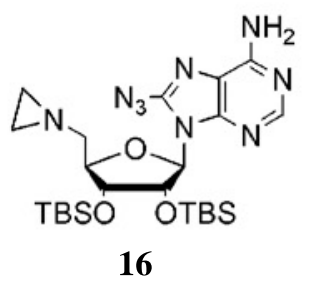



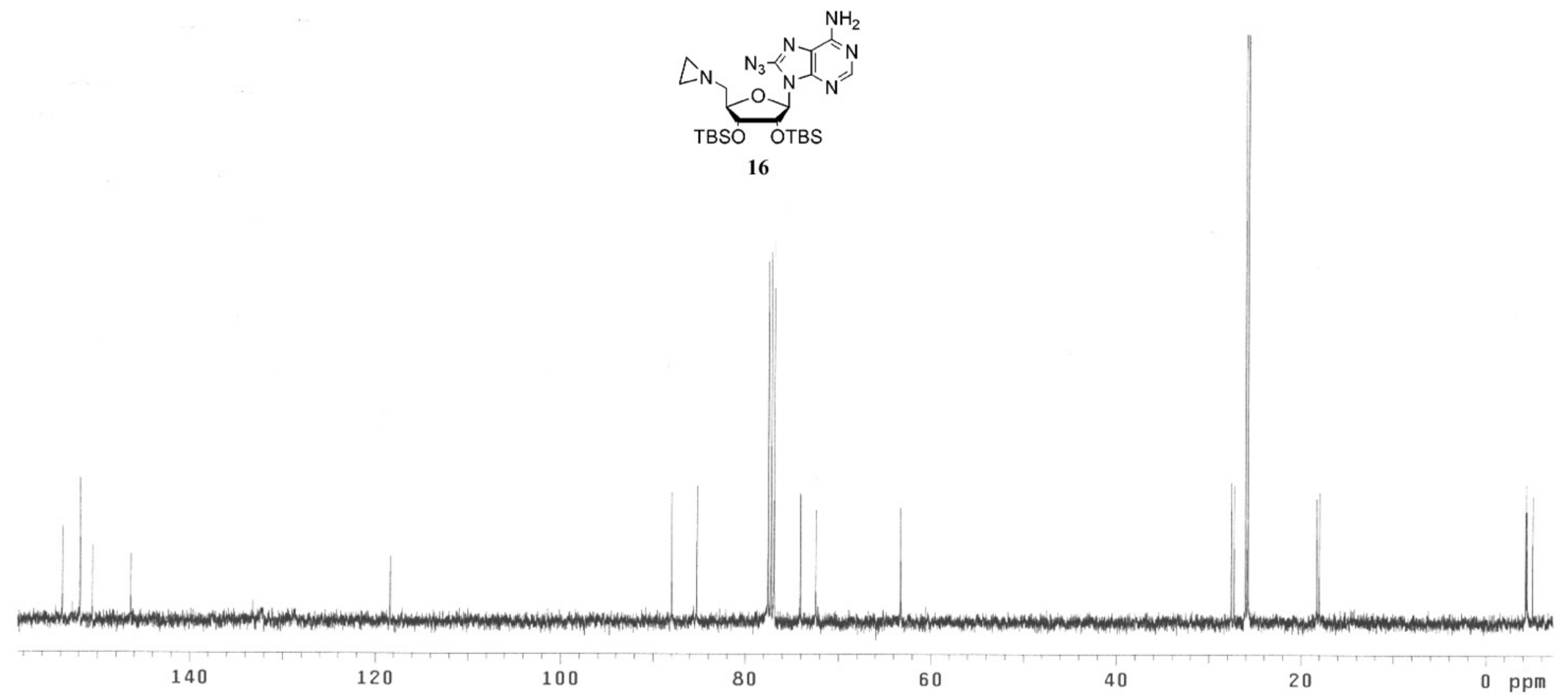


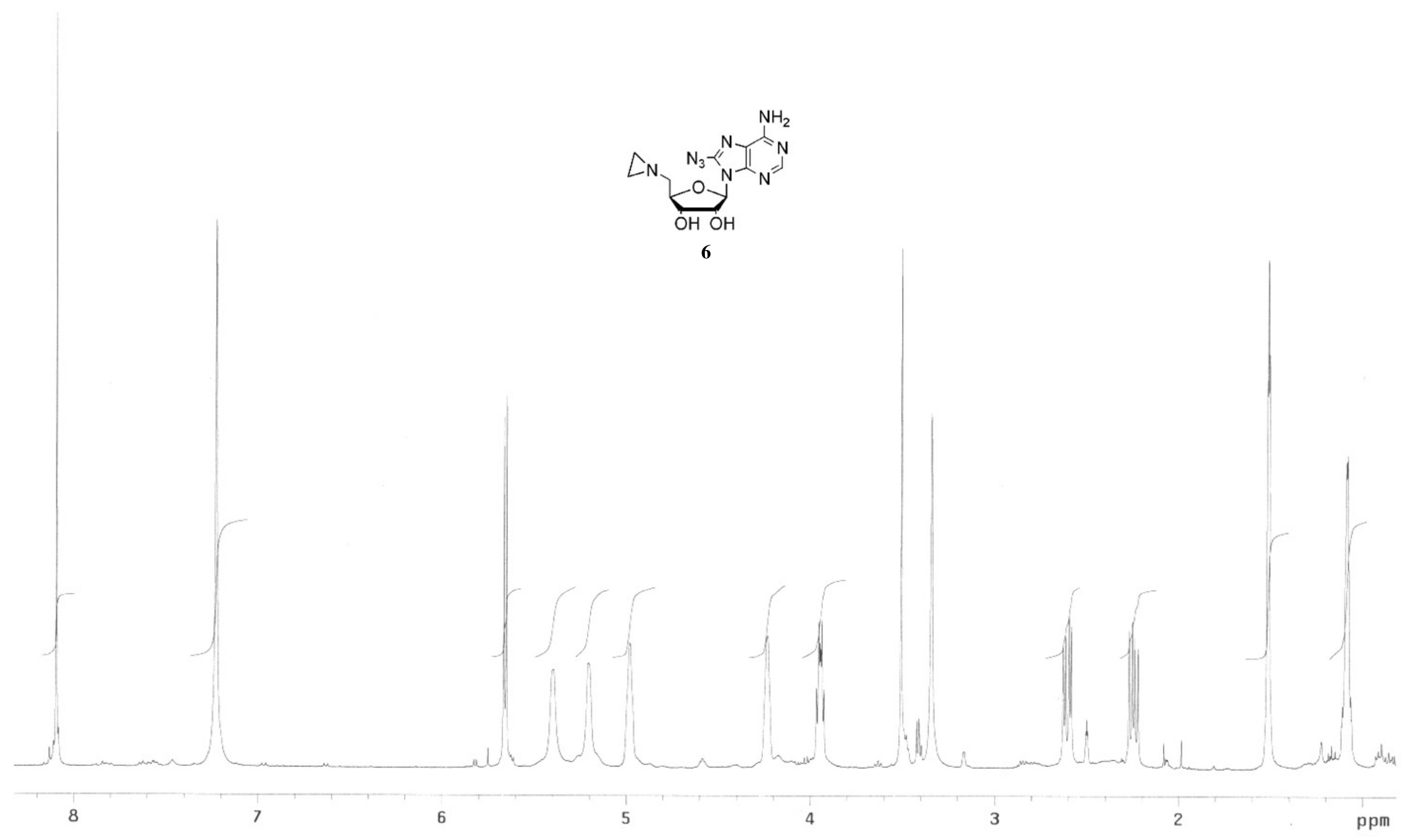



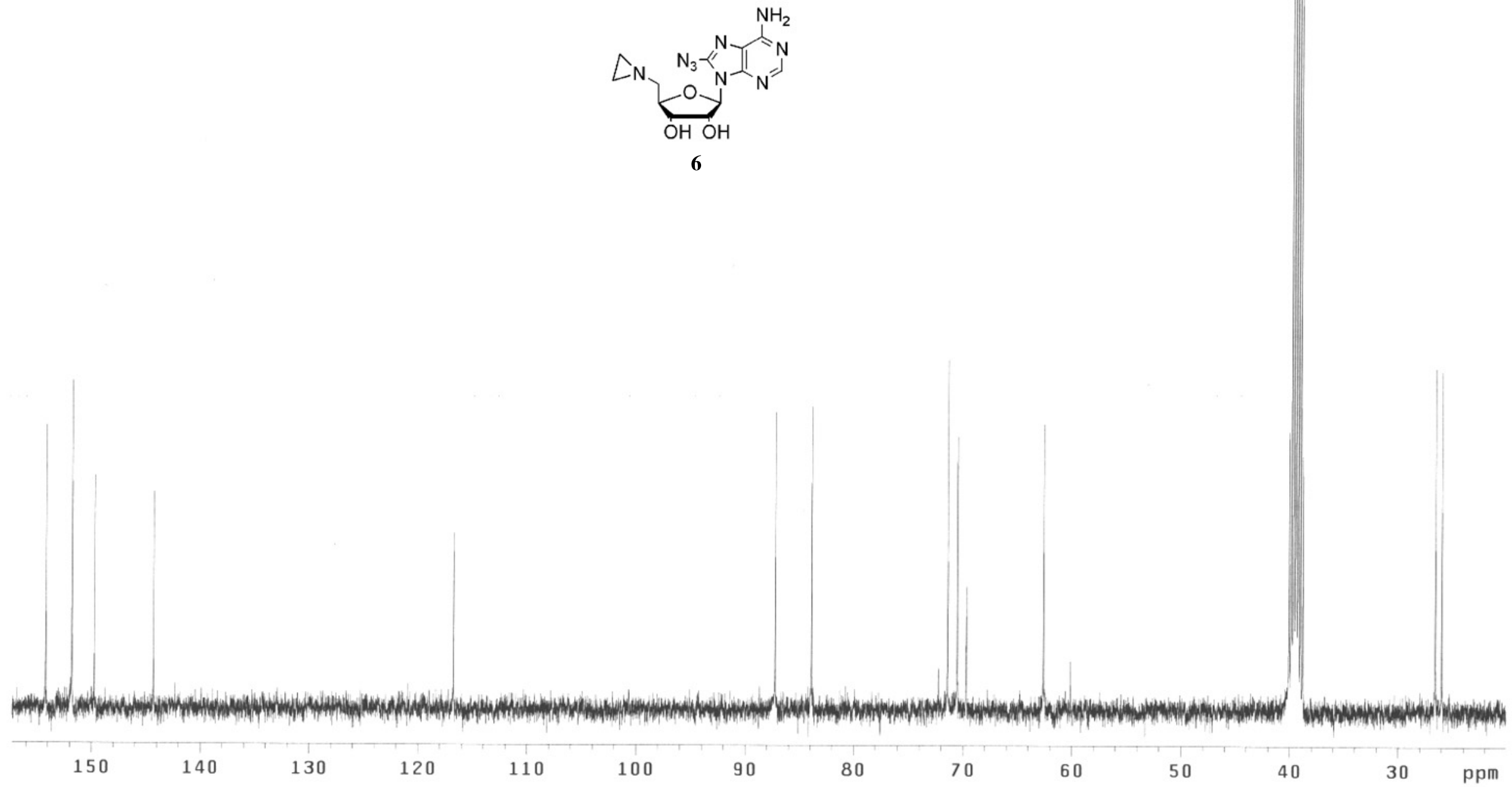